$\delta_{n+1}$ are to be chosen independently of $\delta_{n+2}, \delta_{n+3}, \cdots$ in such manner that the conjugate of the point $w_{n}$ with respect to $R$ lies exterior to the circle $|w|=2^{n+1}$; each $\delta_{n}$ (for $n>1$ ) is subjected then to two conditions, and the numbers $\delta_{n}$ can be determined in succession. The resulting region $R$ is a Jordan region. The sequence $w_{n}$ approaches the boundary point $w=3$ of $R$, and the conjugate of $w_{n}$ with respect to $R$ becomes infinite with $n$. Theorem 3 is established.

HARVARD UNIVERSITY

\title{
ON THE ORDER OF THE PARTIAL SUMS OF FOURIER POWER SERIES ${ }^{1}$
}

\section{OTTO SZÁsZ}

Dedicated to L. Fejér on his sixtieth birthday.

Let $f(x)$ be a Lebesgue integrable function, and denote the partial sums of its Fourier series by $s_{n}(f ; x)$. It is well known that $s_{n}=o(n)$ uniformly ${ }^{2}$ in $x$. Recently W. C. Randels ${ }^{3}$ gave an example showing that this estimate cannot be improved. The same conclusion can be drawn from a note by E. C. Titchmarsh ; ${ }^{4}$ and A. Zygmund in his review of Randels' article (Zentralblatt für Mathematik, vol. 18, p. 353) pointed to another device, using convex coefficient sequences, which would establish the same fact.

In this note a simple construction is given, using a sequence of polynomials in the complex variable $z$. This leads to a sharper result showing that even for Fourier power series (that is, a power series considered on its circle of convergence and integrable) the estimate cannot be improved. Moreover, an example $F(z)=\sum_{n=0}^{\infty} c_{n} z^{n}$ is given which has the additional property that $F(z) /(1-z)$ is a generalized Fourier power series on $|z|=1$.

We start with a sequence of polynomials of increasing degree $P_{n}(z)=\left(\sum_{v=0}^{m} c_{n v} z^{\nu}\right)^{2}=\sum_{v=0}^{2 m} a_{n v} z^{\nu}$ having the following properties:

$$
\frac{1}{2 \pi} \int_{-\pi}^{\pi}\left|P_{n}\left(e^{i x}\right)\right| d x=\sum_{\nu=0}^{m}\left|c_{n \nu}\right|^{2}=1
$$

1 Presented to the Society, April 15, 1939.

${ }^{2}$ In fact, if $c_{0}, c_{1}, \cdots$ are the Fourier coefficients, then $c_{n} \rightarrow 0$. Hence $\sum_{0}^{n}\left|c_{\nu}\right|=o(n)$.

${ }^{3} \mathrm{~W}$. C. Randels, On the order of the partial sums of a Fourier series, this Bulletin, vol. 44 (1938), pp. 286-288.

${ }^{4}$ E. C. Titchmarsh, Principal value Fourier series, Proceedings of the London Mathematical Society, (2), vol. 23 (1925), pp. xli-xliii. 
It is sufficient to consider two special cases, ${ }^{5}$ given in formulas (3) and (4)

$$
P_{n}(z)=(n+1)^{-1}\left(\sum_{0}^{n} z^{\nu}\right)^{2}, \quad n=1,2, \cdots ; m=n .
$$

In this case $\sum_{\nu=0}^{n}\left|c_{n \nu}\right|^{2}=1, \sum_{\nu=0}^{2 n} a_{n \nu}=P_{n}(1)=n+1$, and $\gamma=1$;

(4) $\quad P_{n}(z)=(2 n)^{-1}\left(1-z^{n}\right)^{4}(1-z)^{-2}, \quad n=1,2, \cdots ; m=2 n-1$.

In this case

$$
\begin{aligned}
P_{n}(z) & =(2 n)^{-1}\left\{\left(1-z^{n}\right)^{2}(1-z)^{-1}\right\}^{2} \\
& =(2 n)^{-1}\left\{\left(1+\cdots+z^{n-1}\right)\left(1-z^{n}\right)\right\}^{2} \\
& =(2 n)^{-1}\left(1+\cdots+z^{n-1}-z^{n}-\cdots-z^{2 n-1}\right)^{2} .
\end{aligned}
$$

Hence $\sum_{\nu=0}^{2 n-1}\left|c_{n \nu}\right|^{2}=2 n / 2 n=1$. Furthermore from (4)

$$
P_{n}(z)=(2 n)^{-1}\left(1+2 z+\cdots+n z^{n-1}+\cdots\right) .
$$

Hence

$$
\sum_{\nu=0}^{n-1} a_{n \nu}=\frac{1}{2 n} \cdot \frac{n(n+1)}{2}>\frac{n}{4} ; \quad \gamma=1 / 4 .
$$

Note that in the second example $P_{n}(1)=0$. This property is essential for the last section.

Let $d_{1} \geqq d_{2} \geqq d_{3} \geqq \cdots$. be an arbitrarily given monotone sequence of positive numbers tending to zero, and let $n_{1}<n_{2}<n_{3}<\cdots$ be integers such that $\sum_{\nu=1}^{\infty} d_{n_{\nu}}<\infty$. Denote the degree of the polynomial $P_{n_{\nu}}(z)$ by $2 m_{\nu}$, and let, for example, $\lambda_{1}=1$,

$$
\lambda_{k+1}=2 m_{k}+\lambda_{k}+1, \quad k=1,2, \cdots,
$$

which gives

$$
\lambda_{k+1}=k+1+2 \sum_{\nu=1}^{k} m_{\nu}, \quad k=1,2,3, \cdots .
$$

It is then clear that in the totality of polynomials $z^{\lambda_{\nu}} P_{n_{\nu}}(z)$,

5 The polynomials given in (3) have been used by F. Riesz to construct a Fourier power series for which $\lim \sup _{n \rightarrow \infty} \int_{-\pi}^{\pi}\left|s_{n}\left(e^{i x}\right)\right| d x=\infty$. Cf. A. Zygmund, Proceedings of the London Mathematical Society, (2), vol. 34 (1932), pp. 392-400, and Zygmund, Trigonometrical Series, p. 165. 
$(\nu=1,2, \cdots)$, no power of $z$ is repeated. Hence the series of polynomials

$$
F(z)=\sum_{\nu=1}^{\infty} d_{n_{\nu}} z^{\lambda \nu} P_{n_{\nu}}(z)
$$

with each term written out separately is a power series convergent for $|z|<1$. Moreover, when $0<r<1$,

$$
\frac{1}{2 \pi} \int_{-\pi}^{\pi}\left|F\left(r e^{i x}\right)\right| d x \leqq \frac{1}{2 \pi} \sum_{\nu=1}^{\infty} d_{n_{\nu}} \int_{-\pi}^{\pi}\left|P_{n_{\nu}}\left(r e^{i x}\right)\right| d x=\sum_{\nu=1}^{\infty} d_{n_{\nu}}<\infty ;
$$

whence $F\left(e^{i x}\right)$ exists and is Lebesgue integrable. Note that for both examples the series (7) converges uniformly in any closed region on $|z| \leqq 1$ excluding the point $z=1$. Thus $F(z)$ is continuous in that region.

For example (3) we now have $s_{2 n_{k}+\lambda_{k}}(F ; 1)=\sum_{v=1}^{k}\left(1+n_{v}\right) d_{n_{\nu}}$. Since $d_{n}$ decreases as $n$ increases we get, using (5) and (6),

$$
\begin{aligned}
s_{2 n_{k}+\lambda_{k}}(F ; 1) & \geqq d_{n_{k}}\left(k+\sum_{\nu=1}^{k} n_{\nu}\right)>(1 / 2) d_{n_{k}} \lambda_{k+1} \\
& >(1 / 2) d_{n_{k}}\left(2 n_{k}+\lambda_{k}\right) .
\end{aligned}
$$

Thus $s_{\lambda_{k+1}-1}>(1 / 2)\left(\lambda_{k+1}-1\right) d_{\lambda_{k+1}-1}$, giving

$$
\limsup _{n \rightarrow \infty} \frac{s_{n}}{n d_{n}} \geqq 1 / 2 \text {, }
$$

where $d_{n}$ has been chosen approaching 0 as slowly as we please.

In the case of example (4) we consider

$$
s_{2 m_{k}+\lambda_{k}+n_{k+1}+1}(F ; 1)=(1 / 4) d_{n_{k+1}}\left(1+n_{k+1}\right),
$$

where $m_{k}=2 n_{k}-1$. Thus

$2 m_{k}+1=4 n_{k}-1=\lambda_{k+1}-\lambda_{k}, \quad 1+n_{k+1}=1+\left(\lambda_{k+2}-\lambda_{k+1}+1\right) / 4$.

Also $2 m_{k}+\lambda_{k}+n_{k+1}+1=\lambda_{k+1}+\left(\lambda_{k+2}-\lambda_{k+1}+1\right) / 4$. Denoting this number by $\mu_{k}$, we have $s_{\mu_{k}}>(1 / 4)\left(\mu_{k}-\lambda_{k+1}\right) d_{\mu_{k}}$. Hence

$$
\frac{s_{\mu_{k}}}{\mu_{k} d_{\mu_{k}}}>\frac{1}{4} \frac{\mu_{k}-\lambda_{k+1}}{\mu_{k}}=\frac{1}{4} \frac{\lambda_{k+2}-\lambda_{k+1}+1}{\lambda_{k+2}+3 \lambda_{k+1}+1} .
$$

Assuming, as we may, $\lambda_{k+1} / \lambda_{k} \rightarrow \infty$, we get

$$
\limsup _{n \rightarrow \infty} \frac{s_{n}}{n d_{n}} \geqq 1 / 4 \text {. }
$$


Let us rewrite the function defined by (7) as

$$
F(z)=\sum_{n=1}^{\infty} c_{n} z^{n}
$$

and consider

$$
\int_{0}^{z} F(t) d t=\sum_{n=1}^{\infty} \frac{1}{n+1} c_{n} z^{n+1}=F_{1}(z) .
$$

$F_{1}(z)$ is absolutely continuous. Thus by a theorem of Hardy and Littlewood $\sum_{n=1}^{\infty}\left|c_{n}\right| /(n+1)<\infty$. On the other hand

$$
\begin{aligned}
\sum_{\nu=1}^{n} c_{\nu} & =n \sum_{\nu=1}^{n} \frac{c_{\nu}}{\nu+1}-\sum_{\nu=1}^{n}(n-\nu+1) \frac{c_{\nu}}{\nu+1} \\
& =n s_{n}\left(F_{1} ; 1\right)-\sum_{\nu=1}^{n} s_{\nu}\left(F_{1} ; 1\right) \\
& =n\left\{s_{n}-F_{1}(1)\right\}-\sum_{\nu=1}^{n}\left(s_{\nu}-F_{1}(1)\right) .
\end{aligned}
$$

It now follows that there does not exist a sequence $\left(\delta_{n}\right)$ tending to zero, such that for every absolutely continuous Fourier power series $F(z)$ we have $s_{n}-F_{1}(1)=O\left(\delta_{n}\right)$. For then for a positive number $\rho$ depending on $F$ we should have $\left|s_{n}-F_{1}(1)\right|<\rho \delta_{n},(n=1,2,3, \cdots)$, and hence, from (11),

$$
\left|\sum_{\nu=1}^{n} c_{\nu}\right|<\rho n \delta_{n}+\rho \sum_{\nu=1}^{n} \delta_{\nu}, \quad \frac{1}{n}\left|\sum_{\nu=1}^{n} c_{\nu}\right|<\rho \delta_{n}+\frac{\rho}{n} \sum_{\nu=1}^{n} \delta_{\nu} .
$$

Thus if $\sigma_{n}=2 \max \left(\delta_{n},(1 / n) \sum_{\nu=1}^{n} \delta_{\nu}\right)$, then $\sigma_{n} \rightarrow 0$, and $(1 / n)\left|\sum_{\nu=1}^{n} c_{\nu}\right|$ $<\rho \sigma_{n}$. Let now $d_{n}^{2}=\max _{\nu \geqq n} \sigma_{\nu}$; then $d_{n} \downarrow 0$, and $\left(1 / n d_{n}\right)\left|s_{n}\right|<\rho d_{n} \downarrow 0$, which contradicts the result in (9) and (10).

Now consider again the function $\left(7^{\prime}\right)$ corresponding to the example in (4). Then for $|z|<1,(1-z)^{-1} F(z)=\sum_{n=1}^{\infty} s_{n} z^{n}$, where $s_{n}=\sum_{\nu=1}^{n} c_{\nu}$. From (7)

$$
(1-z)^{-1} F(z)=\sum_{\nu=1}^{\infty} d_{n_{\nu}} z^{\lambda_{\nu}} P_{n_{\nu}}(z)(1-z)^{-1},
$$

where the series converges uniformly in any closed region on $|z| \leqq 1$ excluding the point $z=1$. Now for $0<\epsilon<\pi$

${ }^{6}$ G. H. Hardy and J. E. Littlewood, Some new properties of Fourier constants, Mathematische Annalen, vol. 97 (1926), pp. 159-209, in particular Theorem 16, p. 208. 


$$
\begin{aligned}
\int_{e}^{\pi}\left(1-e^{i x}\right)^{-1} F\left(e^{i x}\right) d x & =\sum_{\nu=1}^{\infty} d_{n_{\nu}} \int_{\epsilon}^{\pi} e^{i \lambda_{\nu} x} P_{n_{\nu}}\left(e^{i x}\right)\left(1-e^{i x}\right)^{-1} d x \\
& =\frac{1}{2} \sum_{\nu=1}^{\infty} d_{n_{\nu}} \frac{1}{n_{\nu}} \int_{\epsilon}^{\pi} e^{i \lambda_{\nu} x}\left(1-e^{i n_{\nu} x}\right)^{4}\left(1-e^{i x}\right)^{-3} d x
\end{aligned}
$$

and

$$
\left(\int_{\epsilon}^{\pi}+\int_{-\pi}^{-\epsilon}\right) e^{i \lambda_{\nu} x}\left(1-e^{i n_{\nu} x}\right)^{4}\left(1-e^{i x}\right)^{-3} d x \rightarrow 0, \quad \epsilon \rightarrow 0 .
$$

Thus $\int_{-\pi}^{\pi}\left(1-e^{i x}\right)^{-1} F\left(e^{i x}\right) d x$ exists as a generalized integral and

$$
\int_{-\pi}^{\pi} F\left(e^{i x}\right)\left(1-e^{i x}\right)^{-1} d x=0 .
$$

On the other hand $s_{1}, s_{2}, s_{3}, \cdots$ are the generalized Fourier coefficients of this function. Thus $o(n)$ is the sharpest estimate for the Fourier coefficients of a generalized Fourier power series. For generalized Fourier series Titchmarsh ${ }^{7}$ constructed an example along essentially different lines.

\section{University of Cincinnati}

7 E. C. Titchmarsh, The order of magnitude of the coefficients in a generalized Fourier series, Proceedings of the London Mathematical Society, (2), vol. 22 (1924), pp. $x x v-x x v i$. 